\title{
Identical Synchronization of a New Chaotic System via Nonlinear Control and Linear Active Control Techniques: A Comparative Analysis
}

\author{
Azizan Bin Saaban ${ }^{1}$, Adyda Binti Ibrahim ${ }^{1}$, Mohammad Shahzad ${ }^{2}$ and Israr Ahmad ${ }^{1,2}$ \\ ${ }^{1}$ School of Quantitative Sciences, College of Arts \& Sciences, UUM, Malaysia \\ ${ }^{2}$ College of Applied Sciences Nizwa, Ministry of Higher Education, \\ Sultanate of Oman
}

azizan@uum.edu.my,adyda@uum.edu.my,shehzad.niz@cas.edu.com,

iak_2000plus@yahoo.com

\section{Abstract}

Most of the synchronization techniques belong to the naster-stave (drive-response) system configurations in which the two chaotic systems are coupled in such a manner that the performance of the second (slave /response) system is influenced by the first (drive/master) system and the first system is not disturbed by the exertion of the second (slave / response) system.

In this research paper, the synchronization problem of two widely used techniques, the Linear Active Control and Nonlinear Control Algorithm have been studied to achieve chaos synchronization of a new chaotic system. In this study, using the Linear Active Control and the Nonlinear Control algorithm and based on the Lyapunov Stability Theory, it has been shown that the two techniques have excellent ransient performance and that analytically as well as graphically, the synchronization is asymptotically globally stable. Numerical simulations are furnished to show the efficiency and effectiveness of the two methods.

Keywords: Synchronization, Limear Active Control, Nonlinear Active Control, Lyapunov, Stability Theory

\section{Introduction}

Chaos has been extensively studied over the past three decades after the pioneering work of Edwards Lorenz on Chaos [1] who revealed that simple three dimensional differential equations with a tiny changes in its initial conditions and parameters can bring major differences in their future states. This extreme sensitive dependence upon initial conditions is known as the "Butterfly Effect" [1]. Mathematically a Chaotic system is a nonlinear determintstic system that displays unpredictable and extremely complex behavior.

Synchonization is one of the most engrossing phenomenan of Chaos. Synchronization of chatic systems is a procedure where two chaotic systems (either equivalent or nonequivalent) adjust a given property of their motion to a common behavior due to coupling or forcing. This ranges from absolute agreement of trajectories to interlocking of phases. This idea of synchronization was first introduced by Pacora and Carroll [2], and since then synchronization of chaotic dynamical systems has received a great deal of interest among scientists from almost all nonlinear sciences for more than the last two decades. Further Chaos Synchronization has many potential applications in different fields such as Secret 
Communications, Laser Physics, Chemical Reactor and in many other biological and physical systems [3-7].

A wide range of techniques have been introduced and are applied successfully to synchronize identical (nearly identical) as well as nonidentical chaotic systems such as Adaptive Control, Backsteeping Method, Active Control Algorithm, Lag Synchronization, Linear Error State Feedback Algorithm, Sliding Mode Control and Nonlinear Active Control Algorithm[8-14], etc.

Recently, the synchronization problem via Active Control and Nonlinear Control Techniques have attracted great interest among the researchers and have been widely accepted as the two powerful techniques used to synchronize two identical as well as nonidentical chaotic systems [8-13].

Chaos Synchronization using Active Control was proposed by E. W. Bai, et al., [8] and has recently been accepted and used as one of the most efficient techniques for synchronizing both identical and nonidentical chaotic systems because of its implementation in practical systems such as, Bonhoffer-van der Pol Oscillators, Windmi and Coullet Systems, Ellipsoidal Satellite and Nonlinear Gyros [7, 16-18], etc. If the nonlinearity of the system is known, an Active Controller can be easily designed according to the given condition of the chaotic system to achieve synchronization globally. There are no derivatives in the controller.

On the other hand, the Nonlinear Control Algorithm is an effective technique for synchronizing two identical as well as nonidentical chaotic systems. Most of the real-world control problems are nonlinear, so Nonlinear Control Technques take the advantage of the given nonlinear system dynamics to produce high-performance designs. No Lyapunov Exponents or Gain Matrix is required forthenr implementation [14]. These features free the designer to focus on the synchronization problem, leaving tiresome analytical calculations.

In reference [19], the authors have proposed and studied a new 3-Dimensional chaotic system by replacing a constant parameter $e$ with a switching function in Qi four-wing attractor and generating an eight-wing chaotic attractor. The new eight-wing chaotic attractor has more complex dynamies and topologically different structures than the original Qi fourwing chaotic attractor.

Motivated from above, the main goal of this research paper is to employ the Linear Active and Nonlinear Active Control Algonthms to study and investigate the global chaos synchronization of identical new 3-D chaotic systems [19]. Based on the Lyapunov Stability Theory [20] and using the approaches in references [12, 14], a class of feedback control schemes will be proposed to achieve the synchronization asymptotically globally. Numerical simulations and graphs yill be furnished to show the effectiveness of the two approaches, then the performance of the two techniques will be compared.

The rest of the paper is organized as follows: In Section 2, the Linear Active Control Methodology has been given and has solved the chaos synchronization problem of a new chaotic system using Linear Active Control Technique. In Section 3, the Nonlinear Control Methodology has been derived and then applied to synchronize a new chaotic system, finally, the concluding remarks are then given in Section 4.

\section{Designing of a Linear Active Controller}

Many synchronization algorithms belong to drive-response (master-slave) system arrangement. The drive-response (master-slave) arrangement means that the two chaotic systems are coupled in such a way that the performance of the second (response/slave) system is influenced by the first (drive/master) system and the first system is not disturbed by the exertion of the second (response/slave) system. 
Consider a drive system described from the following differential equation,

$$
\dot{x}=A_{1} x+h(x)
$$

A response system is defined as,

$$
\dot{y}=A_{2} y+g(y)+\eta(t)
$$

where $\mathbf{x}, \mathbf{y} \in \mathrm{R}^{\mathrm{n}}$ are the state vectors, $A_{1}, A_{2} \in R^{n \times n}$ are constant system matrices and $h(x), g(y): R^{n} \rightarrow R^{n}$ are the nonlinear functions of the corresponding drive-response systems respectively and $\eta(t)$ is the control input. The error dynamics are defined as

$$
\dot{e}_{i}=y_{i}-x_{i}
$$

Thus the error dynamics for synchronization of system (2.1) and (2.2) is described as ,

$$
\dot{e}=\dot{y}-\dot{x}=B e+F(x, y)+\eta(t)
$$

Where, $B=\bar{A}_{2}-\bar{A}_{1}$ is the common parts of the systernatrices in drive-response systems and $F(x, y)=g(y)-h(x)+A_{2} y-A_{1} x+\eta(t)$ contains the nonlinear functions and noncommon terms and $\eta(t)=\left[\eta_{1}(t), \eta_{2}(t), \eta_{3}(t)\right]^{T}$ is the control input injected to the response system.

If $h(x)=g(y)$ and $A_{1}=A_{2}$, then $\mathbf{x}$ and $\mathbf{y}$ are the states of two identical chaotic systems and if $h(x) \neq g(y)$ or $\mathrm{A}_{1} \neq \mathrm{A}_{\text {. }}$, then $\mathbf{x}$ and $y$ are the states of two non-identical chaotic systems.

An appropriate controller ' $\eta(t)$ that satisfies the error system converges to zero,

$$
\text { i.e, } \quad \lim _{t \rightarrow \infty} e_{i}=\lim _{t \rightarrow \infty}\left|y_{i}(t)-x_{i}(t)\right|=0, \forall x, y, e \in R^{n}
$$

Then the two systems (2.1.1) and (2.1.2) are said to be synchronized.

Thus an essential problem in synchronizing two chaotic systems is in the design of a proper Active Controller that eliminates nonlinear terms and non-common parts and contains other parts which achieve asymptotic stability [13] such as,

$$
\eta(t)=-F(x, y)+v(t)
$$

where $v(t)=-k e$ is a linear controller and $k \in R^{n \times n}$ is a feedback constant gain matrix. 
Thus the error dynamics (2.3) becomes,

$$
\dot{e}_{i}=B e+v(t)=B e-k e=(B-k)=M e
$$

where, $M=B-k$.

From equation (2.1.4), if the error system (2.1.4) is a linear system of the form, $\dot{e}=M e$ and if the system matrix $\boldsymbol{M}$ is Hurwitz [21], i.e., all the eigenvalues of the system matrix $\boldsymbol{M}$ are negative, then by the linear control theory [21], the error system will be asymptotically stable, which intimate that the two chaotic systems are synchronized asymptotically globally.

\subsection{Identical Synchronization of a New 3D Chaotic System [18] via Linear Active Control}

System Description: G. Qi, et al., [18] proposed and studied a ney three dimensional autonomous chaotic systems. The differential equation for the new chaotic systems is given as,

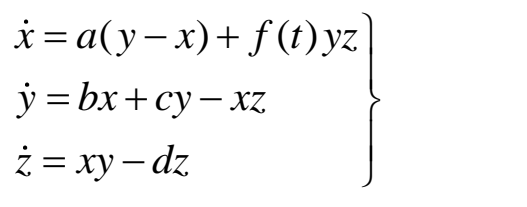

Where $x, y, z \in R^{n \times n}$ are the state variables and $a, b, c$ and $d$ are the system parameters with, $\quad a=14, b=-1 c=16$ and $d=43$ and $f(t)=M \operatorname{sgn}(\sin \omega t)+k$ is a parameter function with ' $\omega$ ' is the switching frequency, $M$ and $k$ are the constant parameters and $t$ is the time.

The authors in eN 18] replaced the parameter ' $e$ ' by sign-switch function and generated an eight-wing chaotic attractor. The new eight-wing chaotic attractor has more complex dynamics and topologically different structures than the original Qi four-wing chaotic attractor. In ref [18], G Qi, and their colleagues have investigated that in the absence of switching parameter (i.e. when $f(t)=1$ ), the system originally shows a four-wing attractor, and when the parameter function switches between the ranges 1 and 19 with frequency $\omega=\frac{2 \pi}{50}$, the system [18] shows an eight-wing chaotic attractor (mother butterfly) and the new four-wing attractor (baby butterfly) is very close to the origin [18]. The analysis of frequency spectra shows that the new (eight-wing chaotic attractor) system has an exceptional broadband frequency bandwidth, which is most advisable for engineering applications such as secrete communications, etc.

To synchronize chaotic system (2.2.1) via Linear Active Control, let us consider the driveresponse systems arrangement, which is described as, 


$$
\left.\begin{array}{l}
\dot{x}_{1}=a\left(y_{1}-x_{1}\right)+f(t) y_{1} z_{1} \\
\dot{y}_{1}=b x_{1}+c y_{1}-x_{1} z_{1} \\
\dot{z}_{1}=x_{1} y_{1}-d z_{1}
\end{array}\right\} \quad \quad \text { (drive system) }
$$

and

$$
\left.\begin{array}{l}
\dot{x}_{2}=a\left(y_{2}-x_{2}\right)+f(t) y_{2} z_{2}+\theta_{1} \\
\dot{y}_{2}=b x_{2}+c y_{2}-x_{2} z_{2}+\theta_{2} \\
\dot{z}_{2}=x_{2} y_{2}-d z_{2}+\theta_{2}
\end{array}\right\}
$$

where $\mathbf{x}_{\mathbf{i}}, \mathbf{y}_{\mathbf{i}}, \mathbf{z}_{\mathbf{i}} \in R^{n}$ for $i=1,2$ are the state variables of the corresponding drive and response systems, $a, b, c$ and $d$ are the system parameters and $\theta(t)=\left[\theta_{1}(t), \theta_{2}(t), \theta_{3}(t)\right]^{T}$ are the feedback controllers which are yet to be designed. The error dynamics of (2.2.2) and (2.2.3) is defined as,

$$
e_{1}=x_{2}-x_{1}, e_{2}=y_{2}-y_{1}, e_{3}=z_{2}-z_{1}
$$

Thus from (2.2.2) and (2.2.3) the error dynamics can be described as;

$$
\left.\begin{array}{l}
\dot{e}_{1}=a\left(e_{2}-e_{1}\right)+f(t)\left(y_{2} z_{2}-y_{1} z_{1}\right)+\theta_{1}(t) \\
\dot{e}_{1}=c e_{2}+b e_{1}-x_{2} z_{2}+\sigma_{1} z_{1}+\theta_{2}(t) \\
\dot{e}_{3}=d e_{3}+x_{2} y_{2}-x_{1} y_{1}+\theta_{3}(t)
\end{array}\right\}
$$

The aim of this section is to design such a feedback Active Controller $\theta(t)=\left[\theta_{1}(t), \theta_{2}(t), \theta_{3}(t)\right]^{T}$ that the error system (2.2.4) convergence to the origin asymptotically, i.e,

$$
\lim _{t \rightarrow \infty}|e(t)|=0, \quad \text { for all } e(0) \in \mathrm{R}^{\mathrm{n}} \text {. }
$$

For this purpose let us re-define the controller $\theta(t)=\left[\theta_{1}(t), \theta_{2}(t), \theta_{3}(t)\right]^{T}$ as,

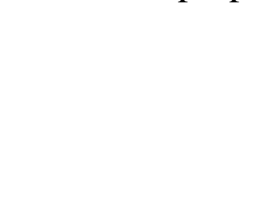

$$
\left.\begin{array}{l}
\theta_{1}(t)=-a e_{2}+f(t)\left(y_{1} z_{1}-y_{2} z_{2}\right)+v_{1}(t) \\
\theta_{2}(t)=-b e_{1}-2 c e_{2}+x_{2} z_{2}-x_{1} z_{1}+v_{2}(t) \\
\theta_{3}(t)=\left(x_{1} y_{1}-x_{2} y_{2}\right)+v_{3}(t)
\end{array}\right\}
$$


Substituting equation (2.2.5) in equation (2.2.4), we have,

Where, $\quad\left(\begin{array}{l}v_{1} \\ v_{2} \\ v_{3}\end{array}\right)=-\left(\begin{array}{lll}k_{11} & k_{12} & k_{12} \\ k_{21} & k_{22} & k_{23} \\ k_{31} & k_{32} & k_{33}\end{array}\right)\left(\begin{array}{l}e_{1} \\ e_{2} \\ e_{3}\end{array}\right)$

The error system (2.2.6) to be controlled is a linear system with a control input $v_{1}, v_{2}$ and $v_{3}$ are functions of $e_{1}, e_{2}$ and $e_{3}$ respectively where the $k_{i j ' s}$ are constant and are known as feedback gains. As long as these feedbacks stabilize the error sys em then $e_{1}, e_{2}$ and $e_{3}$ converge to zero as time 't ' tends to infinity [13]. This imples that the two identical chaotic systems (2.2.2) and (2.2.3) are synchronized asymptotically. Substituting (2.2.7) in (2.2.6), we have

i.e.,

$$
\begin{aligned}
& \left(\begin{array}{l}
\dot{e}_{1} \\
\dot{e}_{2} \\
\dot{e}_{3}
\end{array}\right)=\left(\begin{array}{ccc}
-a & 0 & 0 \\
0 & -c & 0 \\
0 & 0 & -d
\end{array}\right)\left(\begin{array}{l}
e_{1} \\
e_{2} \\
e_{3}
\end{array}\right)-\left(\begin{array}{ccc}
k_{11} & k_{12} & k_{13} \\
k_{21} & k_{22} & k_{23} \\
k_{31} & k_{32} & k_{33}
\end{array}\right)\left(\begin{array}{l}
e_{1} \\
e_{2} \\
e_{3}
\end{array}\right) \\
& \left(\begin{array}{l}
\dot{e}_{1} \\
\dot{e}_{2} \\
\dot{e}_{3}
\end{array}\right)=\left(\begin{array}{ccc}
-a-k_{11} & -k_{12} & k_{13} \\
-k_{21} & -c-k_{22} & -k_{23} \\
-k_{31} & -k_{32} & -d-k_{33}
\end{array}\right)\left(\begin{array}{l}
e_{1} \\
e_{2} \\
e_{3}
\end{array}\right)
\end{aligned}
$$

There are a number of cherces available for the controller coefficient $k_{i j}$ and the choosing of the matrix $\left(\begin{array}{ccc}k_{11} & k_{12} & k_{13} \\ k_{21} & k_{22} & k_{23} \\ k_{3} & k_{32} & k_{33}\end{array}\right)$ should be such that the closed loop system (2.2.6) must have all the eigenvalues with negative real parts so that the error dynamics converges to zero as time $\boldsymbol{t}$ tends to infinity.

For the specific choice of feedback gains;

$$
\left(\begin{array}{lll}
k_{11} & k_{12} & k_{13} \\
k_{21} & k_{22} & k_{23} \\
k_{31} & k_{32} & k_{33}
\end{array}\right)=\left(\begin{array}{ccc}
-11 & 0 & 0 \\
0 & -13 & 0 \\
0 & 0 & -40
\end{array}\right)
$$

With this particular choice, the error system (2.2.8) becomes,

$$
\left(\begin{array}{l}
\dot{e}_{1} \\
\dot{e}_{2} \\
\dot{e}_{3}
\end{array}\right)=\left(\begin{array}{ccc}
-3 & 0 & 0 \\
0 & -3 & 0 \\
0 & 0 & -3
\end{array}\right)\left(\begin{array}{l}
e_{1} \\
e_{2} \\
e_{3}
\end{array}\right)
$$


From equation (2.2.9), It can be seen that the error system (2.2.9) is a linear system of the form, $\dot{e}=M e$. Thus by Linear Control Theory, the system matrix $\boldsymbol{M}$ is Hurwitz [21], and so all the eigenvalues of the system matrix $\boldsymbol{M}$ are negative (-3, -3, -3). Hence the above system (2.2.9) is asymptotically stable, which implies that the two identical systems (2.2.2) and (2.2.3) are synchronized asymptotically globally.

\subsection{Numerical Simulations}

Numerical simulations are furnished to validate the advantages and potency of our proposed method. The parameters for new chaotic system [18] are taken as,

$\mathrm{a}=14, \mathrm{~b}=-1, \mathrm{c}=16 d=43$ and $f(t)=7$, with initial conditions are taken as, $\left(x_{1}(0), y_{1}(0), z_{1}(0)\right)=(8,-12,13)$ and $\left(x_{2}(0), y_{2}(0), z_{2}(0)\right)=(-20,-30,-45)$-where $\mathrm{k}_{1}, \mathrm{k}_{2}$ and $\mathrm{k}_{3}$ are chosen as $(-11,-13,40)$.

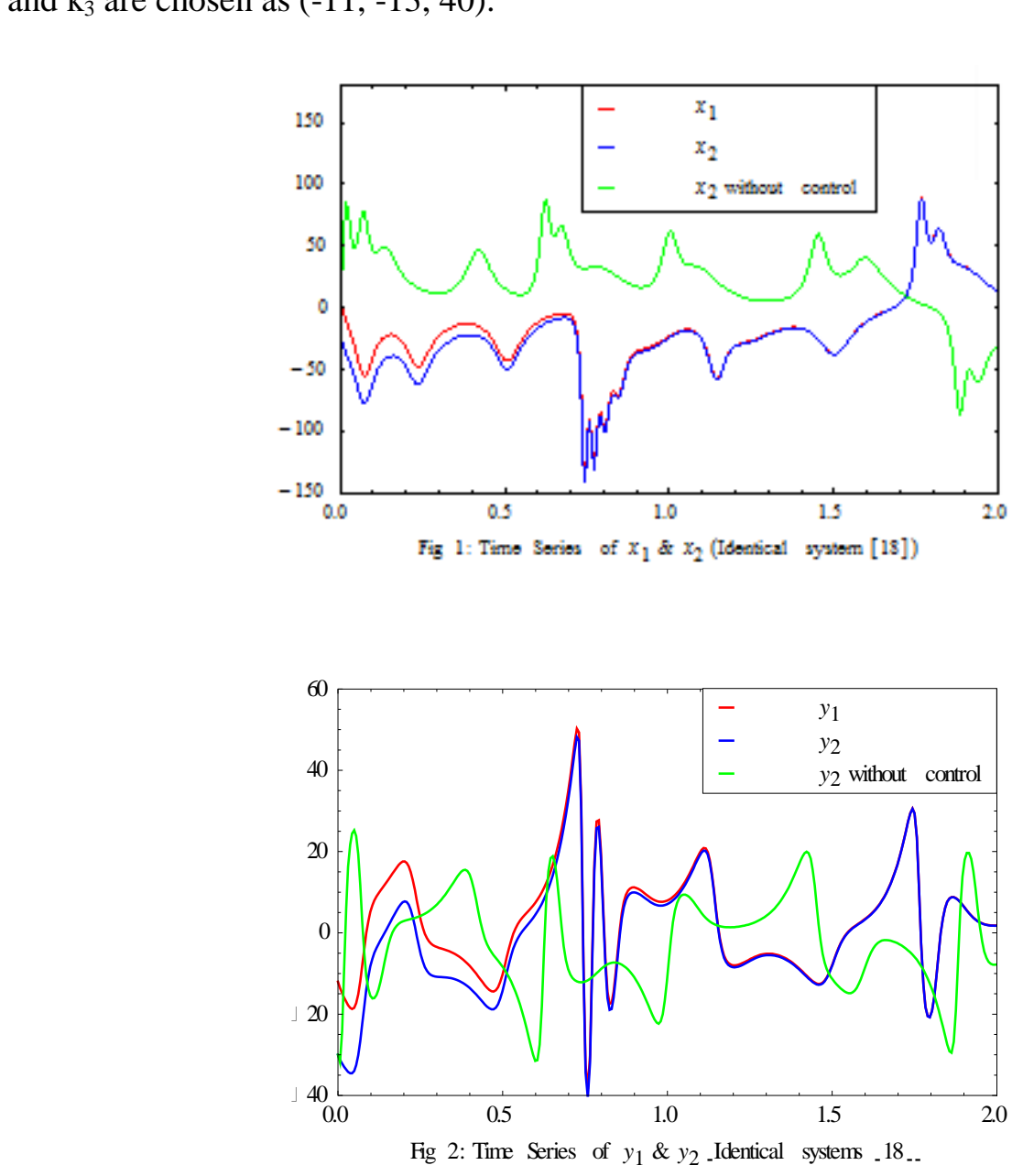



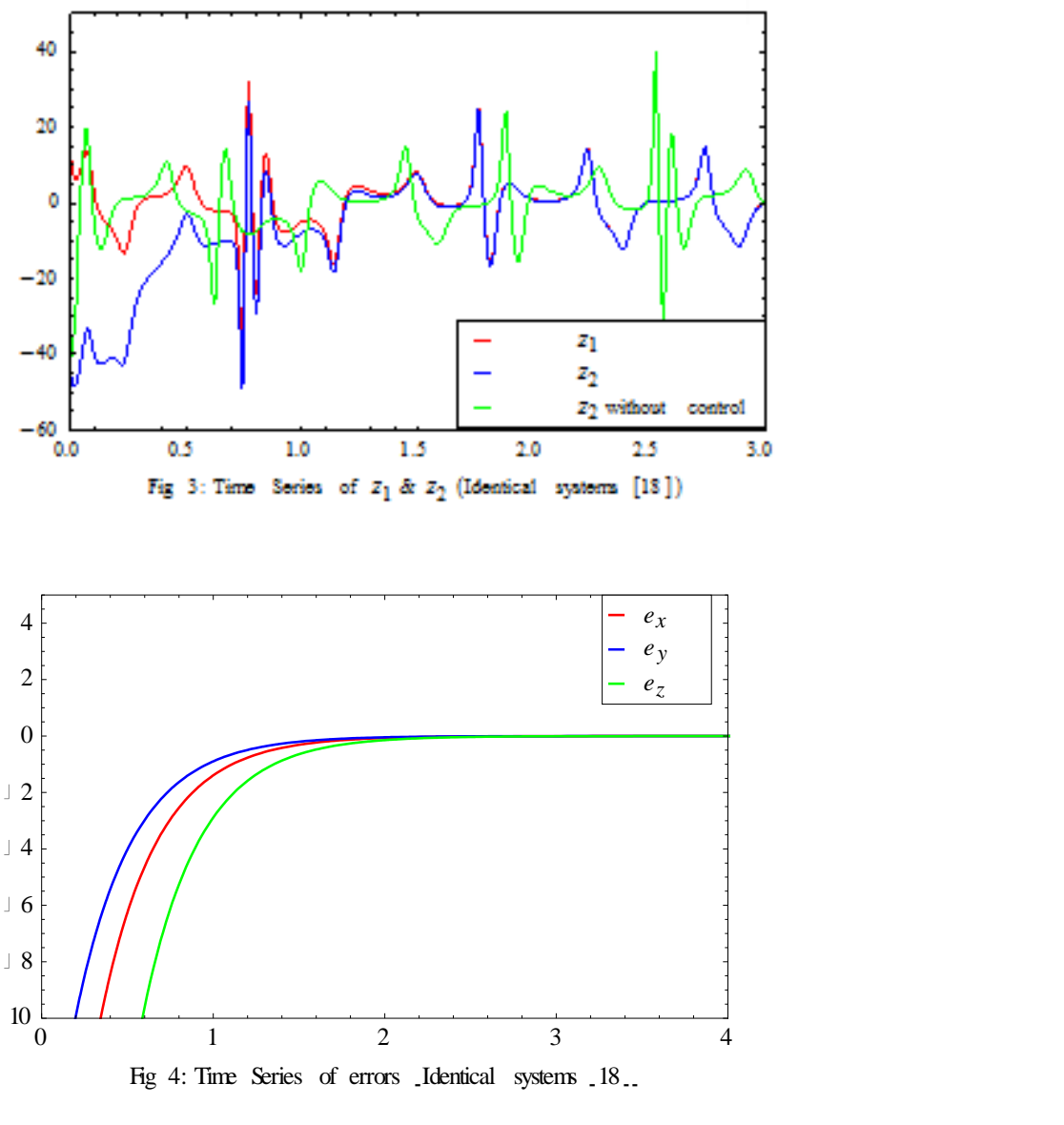

\section{Designing of Nonlinear Controller}

Nonlinear Control Algorithm is an effective approach for synchronization of two identical chaotic systems. No gam matrix or Iyapunov exponents are required for its execution. These characteristios free the designer from monotonous model manipulations to focus on the synchronization problem.

Let us consider a drive-response (master-slave) systems configuration as,

and

$$
x=A_{1} x+g(x) \quad \text { (Drive system ) }
$$

( Response system )

where $x=\left[x_{1}, x_{1}, \ldots, x_{n}\right]^{T}, y=\left[y_{1}, y_{1}, \ldots, y_{n}\right]^{T} \in R^{n \times 1}$ are the corresponding state vectors, $A_{1}, A_{2} \in R^{n \times n}$ are the system matrices, $g, h: R^{n} \rightarrow R^{n}$ are the nonlinear continuous functions of the drive and response systems respectively and $\varepsilon(t)=\left[\varepsilon_{1}, \varepsilon_{2}, \ldots ., \varepsilon_{n}\right]^{T} \in R^{n \times 1}$, is an injected additive nonlinear controller to the controlled system. 
If $g(\square)=h(\square)$ and / or $A_{1}=A_{2}$, then $\mathbf{x}$ and $\mathbf{y}$ are the states of two identical (nearly identical) chaotic systems.

If $g(\square) \neq h(\square)$ and / or $A_{1} \neq A_{2}$, then $\mathbf{x}$ and $\mathbf{y}$ are the states of two nonidentical chaotic systems.

The error dynamics for the synchronization of (3.1) and (3.2) can be described as,

$$
\dot{e}(t)=H(x, y, e)+\varepsilon(t)
$$

where $H(x, y, e)$ the contains linear terms and nonlinear terms of the drive and response systems, where synchronization errors are be defined as;

$$
e_{i}(t)=y_{i}(t)-x_{i}(t)
$$

For the two (identical or non-identical ) chaotic systems withoult controller $(\varepsilon(t)=0$ ), if the initial conditions are $\left(x_{1 d}(0), x_{2 d}(0), \ldots ., x_{n d}(0) \neq y(0), y_{2 r}(0), \ldots y_{n r}(0)\right)$, then the trajectories of the two chaotic systems will quickly diverge from each other in all future time and will become uncorrelated. Hence the role of a feedback controller for the synchronization problem is to restrict the error dynamics converges to zero for allinitial conditions,

i.e.,

$$
\lim _{t \rightarrow \infty}\left\|e_{i}(t)\right\|=\lim _{t \rightarrow \infty} \mid y_{i}(t)-x_{i}(t) \|=0, \quad \text { for all } e_{i}(0) \in \mathrm{R}^{\mathrm{n}}
$$

then the two systems (3.1.1) and (3.1.2) are saictg be synchronized.

Theorem 1. The trajectoriesof the two chaetic (identical/nonidentical) systems for any initial conditions $\left(x_{1 d}(0), x_{2 d}(0), \ldots, x_{n d}(0) \neq y_{1 r}(0), y_{2 r}(0), \ldots ., y_{n r}(0)\right)$ will synchronize

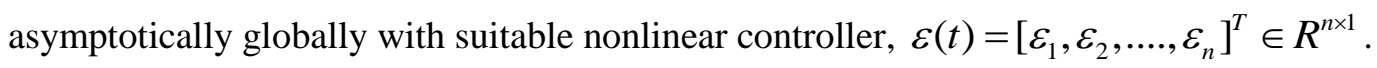

Proof: $\quad$ Let us define a candidate Lyapunov Error Function as,

where the matrix $P=\operatorname{diag}\left(p_{2}, p_{2}, \ldots \ldots ., p_{n}\right)$ is a positive definite matrix [14]. Further it is assumed that all the variables and parameters of the systems are available and measureable.

It may be noticed that, $V: R^{n} \rightarrow R^{n}$ is a positive definite function by construction. It may achieve the synchronization by selecting suitable non-linear controller ' $\varepsilon(t)$ ' to make $\dot{V}(e)=-e^{T} N e$ to be a positive definite matrix (i.e., the matrix $N$ is also a positive definite 
matrix), then by the Lyapunov Stability Theory [20], the states of both drive and response systems will be asymptotically globally synchronized.

\subsection{Identical Synchronization of a New 3D Chaotic System [18] via Nonlinear Active Control}

To synchronize chaotic system (2.2.1) using Nonlinear Control, let us consider the drive-response systems configuration which is described as,

$$
\left.\begin{array}{l}
\dot{x}_{1}=a\left(y_{1}-x_{1}\right)+f(t) y_{1} z_{1} \\
\dot{y}_{1}=b x_{1}+c y_{1}-x_{1} z_{1} \\
\dot{z}_{1}=x_{1} y_{1}-d z_{1}
\end{array}\right\}
$$

(Drive system)

$(3.2 .1)$

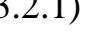

and

$$
\begin{aligned}
& \dot{x}_{2}=a\left(y_{2}-x_{2}\right)+f(t) y_{2} z_{2}+\varepsilon_{1} \\
& \dot{y}_{2}=b x_{2}+c y_{2}-x_{2} z_{2}+\varepsilon_{2} \\
& \dot{z}_{2}=x_{2} y_{2}-d z_{2}+\varepsilon_{2}
\end{aligned}
$$

where $\mathbf{x}_{\mathbf{i}}, \mathbf{y}_{\mathbf{i}}, \mathbf{z}_{\mathbf{i}} \in R^{n}$ for $i=1,2$ are the statefyariables of he corresponding drive and response systems, $a, b, c$ and $d$ are the system parameters and $\varepsilon(t)=\left[\varepsilon_{1}(t), \varepsilon_{2}(t), \varepsilon_{3}(t)\right]^{T}$ are the non-linear controllers which have to be designed. The error dynamics of (3.2.1) and (3.2.2) are defined as,

$$
e_{1}=x_{2}-x_{1}, e_{2}=y_{2}-y_{1}, e_{3}=z_{2}-z_{1}
$$

Thus from (3.2.1) and (3.2.1) the error dynamics can be described as;

$$
\left.\begin{array}{l}
e_{1}=c e_{2}+b e_{1}-e_{2} z_{2}+x_{1} z_{1}+\varepsilon_{2} \\
\dot{e}_{3}=-d e_{3}+x_{2} y_{2}-x_{1} y_{1}+\varepsilon_{3}
\end{array}\right\}
$$

The aim of the synchronization problem is to design a feedback controller $\varepsilon(t)=\left[\varepsilon_{1}(t), \varepsilon_{2}(t), \varepsilon_{3}(t)\right]^{T}$ such as,

$$
\lim _{t \rightarrow \infty}\|e(t)\|=0, \quad \text { for all } e(0) \in R^{n}
$$

The main objective of this section is to investigate and study the synchronization of two identical chaotic systems (3.2.1) and (3.2.2) by designing such a feedback controller that the error dynamics of the two identical chaotic systems convergence to the origin asymptotical 
globally with less control effort and sufficient transient speed. For these motivations, we assume the following theorem.

Theorem 2. The trajectories of the two chaotic Systems (3.2.1) and (3.2.2) will achieve asymptotically globally synchronization for initial conditions, $\left(x_{d}(0), y_{d}(0), z_{d}(0)\right) \neq\left(x_{r}(0), y_{r}(0), z_{r}(0)\right)$ with following control law:

$$
\begin{aligned}
& \varepsilon_{1}=-a e_{2}-f(t)\left(y_{2} z_{2}-y_{1} z_{1}\right) \\
& \varepsilon_{2}=-2 c e_{2}-b e_{1}+x_{2} z_{2}-x_{1} z_{1} \\
& \varepsilon_{3}=x_{1} y_{1}-x_{2} y_{2}
\end{aligned}
$$

Proof: Let us construct a Lyapunov error function candidate as;

$$
V(e)=e^{T} P e
$$

where $P=\left(\begin{array}{ccc}0.5 & 0 & 0 \\ 0 & 0.5 & 0 \\ 0 & 0 & 0.5\end{array}\right)$ is a positive definite function.

Now the time derivative of the Lyapunov error function is,

$$
\dot{V}(e)=-a e^{2}-e_{2}^{2}-d e^{2}=e^{T}\left(\begin{array}{ccc}
14 & 0 & 0 \\
0 & 16 & 0 \\
0 & 0 & 43
\end{array}\right) e \prec 0
$$

Hence based on Lyapunov Stability Theory [20], the origin of the error dynamics of two identical chaotic systems converge to the origin asymptotically. Thus the two chaotic systems (3.2.1) and (3.2.2) are asymptotically globally synchronized. 
International Journal of Hybrid Information Technology Vol.7, No.1 (2014)
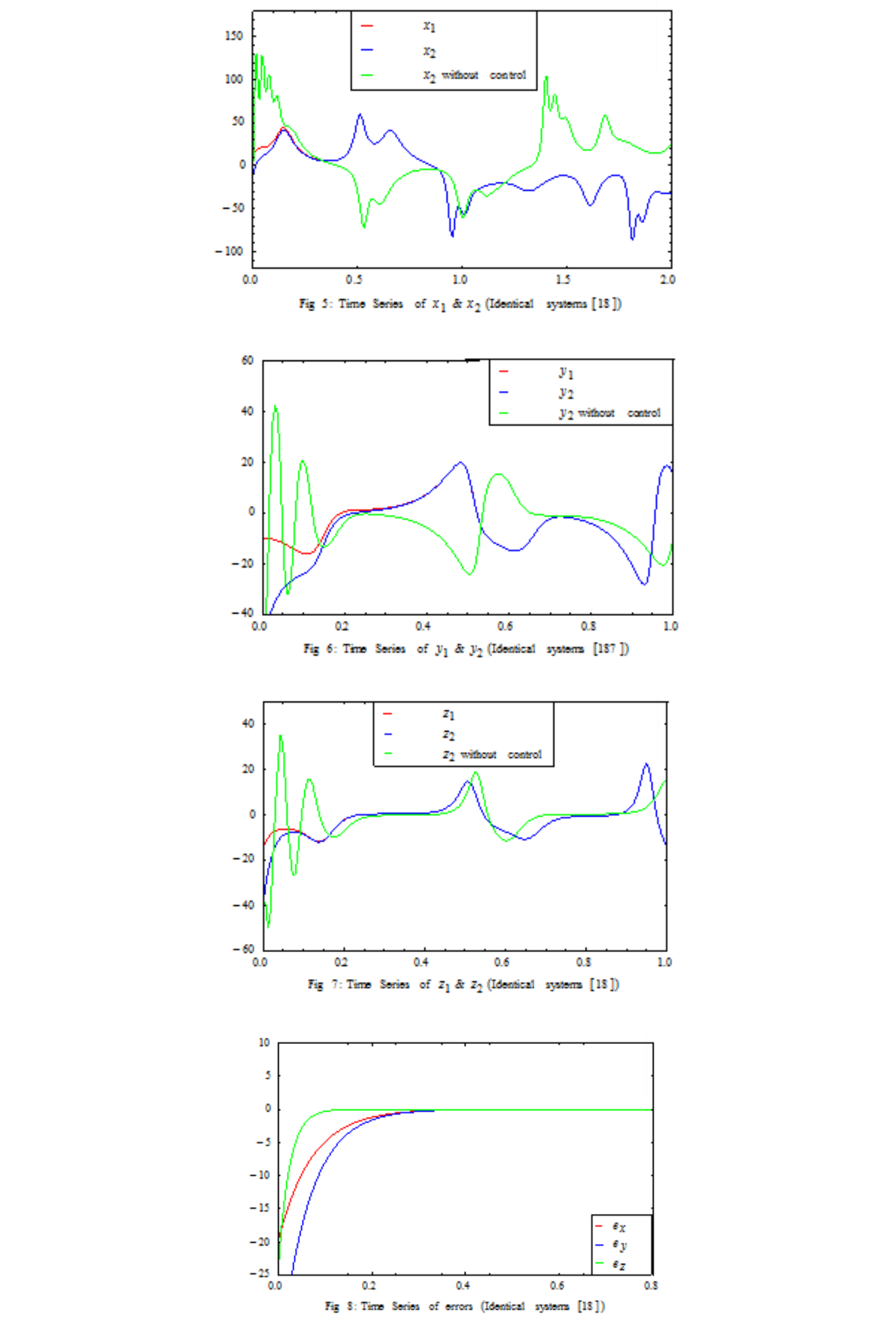


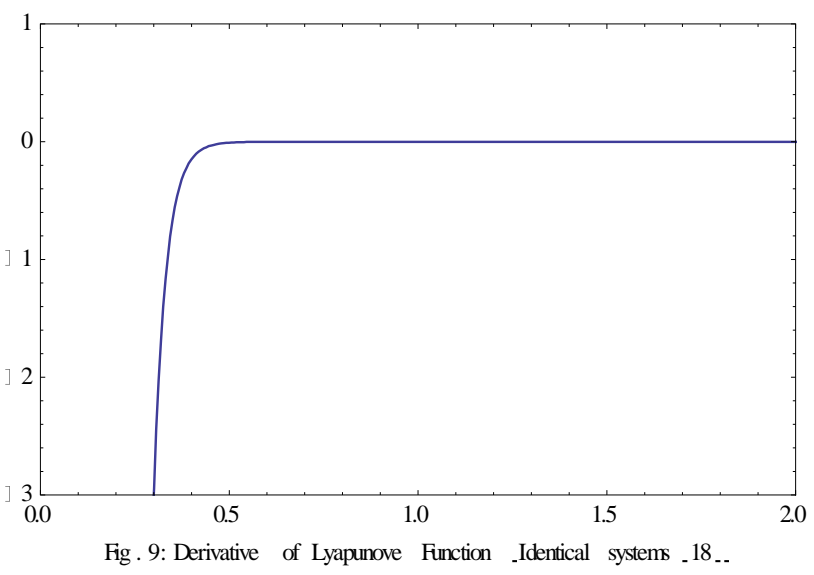

\subsection{Numerical Results}

Numerical simulations are presented to verify the effectiveness of the proposed method. The parameters for the new chaotic system [18] are selected as, $a=14, \mathrm{~b}=-1, \mathrm{c}=16$ $d=43$ and $f(t)=7$, with initial conditions are taken as, $x_{1}(0), y_{1}(0, z,(0))=(10,-10,-15)$ and $\left(x_{2}(0), y_{2}(0), z_{2}(0)\right)=(-25,-50,-40)$.

\section{Conclusion}

In this paper, chaos synchronization of the unified chaotic system has been investigated using Linear Active Control and Nonlinear Active Control. Based on Lyapunov Stability Theory and Ruth-Hurwitz Criterion, suitable feedback controllers are designed to achieve synchronization asymptotically globally: IChas been noticed that the two controllers have exceptional transient performances. Results are presented in graphical forms with time history.

The states of the two unified chaotic systems synchronized are shown in Figures 1-3 and 57 and the stability of the errors dynamics are shown in Figure 4 and 8 while figure 9 shows the derivative of the LyapunovError Function for identical chaotic systems.

However, from the complexities of designing Linear Active Controllers (2.2.5), we can see that major control efforts were required to synchronize two identical chaotic systems. The choice of the high gain coefficients may increase the transient speed but on the other hand, internal resonances may occur which may create large vibrations within the system that in turn can excite the system resulting in the synchronization being disregarded completely. Hence the complications of choosing the proper gain matrix using Linear Active Controller is still unsolved

On the other hand, using the Nonlinear Control Technique, there is no need to find Lyapunov exponent or to construct a gain matrix. These characteristics, freeing the designer from model manipulations, and allowing him to focus on the synchronization problem would be helpful in practical applications. Numerical simulations showed that using the Nonlinear Control, the error dynamics converges to the equilibrium point smoothly with a faster rate (Figure 4 and 8) than using Active Controller.

It is worthwhile mentioning that Nonlinear Control Techniques can under certain conditions have the priority over the Active Control technique from both the stability and analytically problems standpoint. 


\section{Acknowledgements}

We extend our gratitude to Mr. Frances A. Andrew who proof-read this manuscript and suggested many worthwhile changes to improve on its style and grammatically. Mr. Frances A. Andrew who I Andrew is a lecturer in English at College of Applied Sciences, Nizwa, Ministry of Higher education, Sultanate of Oman. He is also a published author of science fiction novels.

\section{References}

[1] E. Lorenz, "Deterministic nonperiodic flow", Journal of the Atmospheric Sciences, vol. 20, no. 2, (1963).

[2] L. Pecora and T. Carroll, "Synchronization in Chaotic Systems", Phys. Rev. Lett., vol. 64, (1990).

[3] O. Moskalenko, et al., "Generalized synchronization of chaos for secure communication: Remarkable stability to noise", Phys. Lett. A., vol. 374, (2010).

[4] A. N. Pisarchik, et al., "Synchronization of Shilnikov Chaos in a $\mathrm{CO}_{2}$ Laser with feedback", Laser Physics, vol. 11, no. 11, (2001).

[5] K. Miyakawa, T. Okabe, M. Mizoguchi and F. Sakamoto, "Synchronization in the discrete chemical oscillation system", J. Chem. Phys., vol. 103, (1995).

[6] M. G. Rosenblum, et al., "Synchronization approach to analysis of Biological systems", An Interdisciplinary Sci. Journal on Random Processes in Physical, Biological and Technological Systems, vol. 4, Issue 01, (2004).

[7] A. N. Njah and U. E. Vincent, "Synchronization and Anti-synchronization of chaos in an extended Bonho ffer-van der Pol Oscillator using Active control”, Journal of Sound and Vibrations, vol. 319, (2009).

[8] E. W. Bai and K. E. Lonngren, "Synchronization of two Lorenz system using Active control", Phy. Rev. Lett., vol. 64, (1997).

[9] S. Vaidyanathan, "Adaptive Control and Synchronization and Synchronization of Shimuzu-Morioka Chaotic systems”, IJFCST, vol. 2, no. 4, (2012).

[10] E. Vincent, "Chaos synchronization using Active control and Backsteeping Control", Nonlinear Any: Modelling \& Control, vol. 13, no. 2, (2008).

[11] G. P. Jiang, et al., "A global synchronization criterion for coupled chaotic systems via unidirectional linear error state feedback approach", Int. J. Bifurcation Chaos, vol. 12, no. 10, (2002).

[12] M. Akbar and U. Ozguner, "Decenterilization Sliding Mode Control Design using overlapping decomposition”, Autometica, vol. 38, (2002).

[13] V. Sundarapandian, "Hybrid Synchronization of Liu Chen and Tigan systems by Active Nonlinear Control", International Journal of Advances in Science and Technology, vol. 3, no. 2, (2011).

[15] G. Y. Qi, et al., "Generation of an eight-wing chaotic attractor from QI-3D four-wing chaotic system", International J. Bifurcation and Chaos, vol 22, no. 12, (2012).

[16] S. Vaidyanathan and S. Rasappan, Global Chaos Sycnhronization for WNDMI and COULLET Chaotic systems using Acive Control”, IJE\&T(JCET), vol. 3, issue 2, (2013).

[17] M. Shehzaa, "Chaos Synchronization of an Ellipsoidal Satellite via Active Control”, Prog. in Applied Mathematics, vol 3, no. 2, (2012)

[18] Y. lei, W. Xu and H. Zheng, "Synchronization of two chaotic nonlinear Gyros using active control", Phys. lett. A, vol. 343, (2005),pp. 153-158.

[19] G. Y. Qi, et al., "Genertion of an eight-wing chaotic attractor from QI-3D four-wing chaotic system", International J. Bifurcation and Chaos, vol. 22, no. 12, (2012).

[20] H. K. Khalil, "Non Linear dynamical Systems", Prentice Hall, ( $3^{\text {rd }}$ edi.), NJ 07458, USA, (2002).

[21] R. C. Dôf and R. H. Bishop, "Modern Control Systems", 9th Ed. Pricton Hall, (2001).

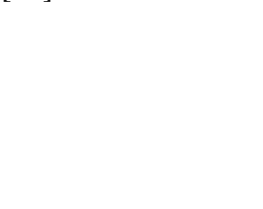

